\title{
SEMI-UNIFORM SUB-ADDITIVE ERGODIC THEOREMS FOR DISCONTINUOUS SKEW-PRODUCT TRANSFORMATIONS
}

\author{
MEIRONG ZHANG, ZUOHUAN ZHENG, AND ZHE ZHOU
}

(Communicated by Yingfei Yi)

\begin{abstract}
In this paper we will establish some semi-uniform ergodic theorems for skew-product transformations with discontinuity from the point of view of topology. The main assumptions are that the discontinuity sets of transformations are neglected in some measure-theoretical sense. The theorems have extended the classical results which have been established for continuous dynamical systems.
\end{abstract}

\section{INTRODUCTION}

Generally speaking, models from classical mechanics will lead to topological or differentiable dynamical systems. One of the typical features is that these systems are continuous in temporal and spatial variables. When the phase space of a dynamical system is compact, the celebrated Bogoliubov-Krylov theorem (see, for example, [8]) asserts that such a system always admits some invariant Borel probability measures and, consequently, can be studied using various ergodic theorems.

The strongest ergodic theorem is the celebrated Oxtoby ergodic theorem [11, which is also called the unique ergodic theorem [8, 14]. For discrete topological dynamical systems, this reads as follows. Suppose that $\Phi: X \rightarrow X$ is a continuous mapping on a compact metric space $X$ so that $\Phi$ admits the unique invariant Borel probability measure $\mu$. Then, for any $f \in C(X)=C(X, \mathbb{R})$,

$$
\lim _{n \rightarrow+\infty} \frac{1}{n} \sum_{i=0}^{n-1} f\left(\Phi^{i}(x)\right)=\int_{X} f \mathrm{~d} \mu
$$

uniformly in $x \in X$.

However, a general dynamical system $\Phi: X \rightarrow X$ will admit many invariant Borel probability measures, and result (1.1) fails in general. A generalization of (1.1) is given by Johnson and Moser [7]. In the discrete-time case, the result reads as follows. Suppose that $\Phi: X \rightarrow X$ is a continuous mapping on a compact metric space $X$. Moreover, $h: X \rightarrow \mathbb{R}$ is a continuous function such that

$$
\int_{X} h \mathrm{~d} \mu=a
$$

Received by the editors January 26, 2011 and, in revised form, November 29, 2011.

2010 Mathematics Subject Classification. Primary 37A20; Secondary 28A35, 58D05.

Key words and phrases. Skew-product transformation, invariant Borel probability measure, semi-uniform convergence, ergodic theorem, sub-additive process. 
for all invariant Borel probability measures $\mu$ of $\Phi$, where $a \in \mathbb{R}$ is a constant independent of $\mu$. Then

$$
\lim _{n \rightarrow+\infty} \frac{1}{n} \sum_{i=0}^{n-1} h\left(\Phi^{i}(x)\right)=a
$$

uniformly in $x \in X$. This is usually called the uniform ergodic theorem. A nice application is illustrated by the definition of the rotation number of the linear Schrödinger equation with an almost periodic potential [7].

In the study of strange compact invariant sets of quasi-periodically forced systems, some semi-uniform ergodic theorems have been established for topological dynamical systems. See [12, 13, 18. In order to study rotation numbers of a forced orientation-preserving measurable transformation of circle, a uniform almostadditive ergodic theorem has been given in [4].

Recently, the dynamics of linear Schrödinger equations with almost periodic potentials and with phase transitions over periodic or almost periodic lattices have been studied in [10, 16, 17. It is found that these will lead to 'dynamical systems' which admit both temporal and spatial discontinuity [17. Note that general systems with (spatial) discontinuity may not admit any invariant Borel probability measure, and, consequently, any ergodic theorem [6, 14] is not applicable. However, based on the skew-product structure of the induced systems of the above problems, Zhang and Zhou have given in [17 the complete extensions of the Bogoliubov-Krylov theorem and the Johnson-Moser uniform ergodic theorem to the so-called skewproduct quasi-flows which admit both temporal and spatial discontinuity, under some measure-theoretical and topological assumptions on the (spatial) discontinuity sets of these systems.

Suggested by those results in [17, the aim of this paper is to give some complete extensions of the semi-uniform ergodic theorems to skew-product transformations (SPT), defined as follows.

Definition 1.1. Suppose that $\Omega$ and $X$ are compact metric spaces, while $\phi: \Omega \rightarrow \Omega$ is a continuous transformation. We say that $\Phi: \Omega \times X \rightarrow \Omega \times X$ is a skew-product transformation with the base $\phi$, abbr. as SPT, if

- there holds $\pi \circ \Phi=\phi \circ \pi$ where $\pi: \Omega \times X \rightarrow \Omega$ is the projection and

- $\Phi: \Omega \times X \rightarrow \Omega \times X$ is Borel measurable.

Such a definition is motivated by the problems in [17]. One of the important features is that it allows the discontinuity of $\Phi(\omega, x)$ in the phase space $\Omega \times X$.

Our semi-uniform ergodic theorems for SPT will be proved in two cases: additivity and sub-additivity. Of course, some restrictions on the discontinuity of SPT $\Phi$ is necessary in these theorems. To describe these, denote

$$
D_{\Phi}:=\{(\omega, x) \in \Omega \times X: \Phi \text { is discontinuous at }(\omega, x)\} .
$$

Since $\phi: \Omega \rightarrow \Omega$ is continuous, the set $\mathcal{M}(\Omega, \phi)$ of $\phi$-invariant Borel probability measures on $\Omega$ is non-empty. The restriction on discontinuity of $\Phi$ is as follows:

$$
\nu\left(\pi\left(D_{\Phi}\right)\right)=0 \quad \forall \nu \in \mathcal{M}(\Omega, \phi) .
$$

Note that $\nu\left(\pi\left(D_{\Phi}\right)\right)$ does make sense; the reason is stated after Theorem 2.3 in Section 2. Such a hypothesis has been used in [17] to obtain the extended BogoliubovKrylov theorem for skew-product quasi-flows. In fact, if an SPT $\Phi$ satisfies $\left(H_{1}\right)$, then $\Phi$ admits some invariant Borel probability measures: $\mathcal{M}(\Omega \times X, \Phi) \neq \emptyset$. Note that $\left(H_{1}\right)$ is some measure-theoretical assumption on discontinuity of $\Phi$. 
The semi-uniform ergodic theorem for the additive case that we will obtain is as follows.

Theorem 1.2. Let $\Phi$ be an SPT on $\Omega \times X$ with the base $\phi$ on $\Omega$ fulfilling $\left(H_{1}\right)$. Suppose that $a \in \mathbb{R}$ and $h \in C(\Omega \times X)$ satisfying

$$
\int_{\Omega \times X} h \mathrm{~d} \mu \leq a \quad \forall \mu \in \mathcal{M}(\Omega \times X, \Phi) .
$$

Then, for any given $\varepsilon>0$, there exists some $N_{\varepsilon} \in \mathbb{N}$ such that for all $n \geq N_{\varepsilon}$, there holds

$$
\frac{1}{n} \sum_{i=0}^{n-1} h\left(\Phi^{i}(\omega, x)\right) \leq a+\varepsilon \quad \forall(\omega, x) \in \Omega \times X .
$$

The proof of Theorem 1.2 will be given in Section 3. An example of SPTs fulfilling $\left(H_{1}\right)$ can be given by the following linear Schrödinger equations with fixed phase transitions:

$$
\begin{cases}y^{\prime \prime}+Q(t) y=0 & \text { for } t \in \mathbb{R} \backslash \Gamma, \\
\left(\begin{array}{c}
y\left(t_{i}+\right) \\
y^{\prime}\left(t_{i}+\right)
\end{array}\right)=R_{\alpha}\left(\begin{array}{c}
y\left(t_{i}-\right) \\
y^{\prime}\left(t_{i}-\right)
\end{array}\right) & \text { at } t_{i} \in \Gamma,\end{cases}
$$

where $Q: \mathbb{R} \rightarrow \mathbb{R}$ is an almost periodic potential, $Q \in \mathcal{C}_{\text {ap }}, \Gamma=\left\{t_{i}\right\}_{i \in \mathbb{Z}} \subset \mathbb{R}$ is an almost periodic lattice, $\Gamma \in \mathcal{L}_{\text {ap }}$, and $R_{\alpha}$ is a rigid rotation with argument $\alpha$. In the polar coordinates $y=r \sin \theta, y^{\prime}=r \cos \theta$, arguments $\theta(t)$ of (1.4) satisfy

$$
\begin{cases}\theta^{\prime}=\cos ^{2} \theta+Q(t) \sin ^{2} \theta & \text { for } t \in \mathbb{R} \backslash \Gamma, \\ \theta\left(t_{i}\right)=\theta\left(t_{i}-\right)+\alpha & \text { at } t_{i} \in \Gamma .\end{cases}
$$

Here the argument is understood to be right-continuous in time $t \in \mathbb{R}$, i.e., $\theta(t)=$ $\theta(t+)$. Denote the translation by

$$
\phi(Q, \Gamma):=\left(Q(\cdot+1),\left\{t_{i}-1\right\}_{i \in \mathbb{Z}}\right), \quad(Q, \Gamma) \in \mathcal{C}_{\text {ap }} \times \mathcal{L}_{\text {ap }} .
$$

As in [7, 16], we embed (1.5) into a family of equations

$$
\begin{cases}\theta^{\prime}=\cos ^{2} \theta+q(t) \sin ^{2} \theta & \text { for } t \in \mathbb{R} \backslash \gamma \\ \theta\left(t_{i}\right)=\theta\left(t_{i}-\right)+\alpha & \text { at } t_{i} \in \gamma\end{cases}
$$

where $(q, \gamma)$ runs over the discrete hull $E_{1}(Q, \Gamma)$ defined by

$$
E_{1}(Q, \Gamma):=\operatorname{closure}_{\left(\mathcal{C}_{\text {ap }} \times \mathcal{L}_{\text {ap }}, d\right)}\left\{\phi^{k}(Q, \Gamma): k \in \mathbb{Z}\right\} .
$$

We can check that $E_{1}(Q, \Gamma)$ is a compact Abelian group and the translation $\phi$ has a unique invariant Borel probability measure being the Haar measure $\nu_{0}$ of $E_{1}(Q, \Gamma)$ : $\mathcal{M}\left(E_{1}(Q, \Gamma), \phi\right)=\nu_{0}$. Let us denote the solution of (1.6) with the initial value $\theta_{\alpha}(0 ; q, \gamma, \vartheta)=\vartheta \in \mathbb{R}$ by $\theta_{\alpha}(t ; q, \gamma, \vartheta) \in \mathbb{R}$. Since the vector field of (1.6) is $2 \pi$ periodic in $\theta$, (1.6) can be considered on the circle $\mathbb{S}_{2 \pi}:=\mathbb{R} / 2 \pi \mathbb{Z}$. Now we can construct a skew-product transformation by

$$
\Phi(q, \gamma, \vartheta):=\left(\phi(q, \gamma), \theta_{\alpha}(1 ; q, \gamma, \vartheta)\right), \quad(q, \gamma, \vartheta) \in E_{1}(Q, \Gamma) \times \mathbb{S}_{2 \pi} .
$$

It can be verified that $\Phi$ is an SPT fulfilling $\left(H_{1}\right)$. For the detailed argument, see [17.

To define the Lyapunov exponents, the celebrated Kingman sub-additive ergodic theorem [2, 9] is important. In this paper, we will establish some semi-uniform subadditive ergodic theorems for SPTs from the point of view of topology. 
Definition 1.3. Suppose that $T: Y \rightarrow Y$ is a measurable mapping. A sequence $\left\{\varphi_{n}\right\}_{n \in \mathbb{N}}$ of continuous functions $\varphi_{n}: Y \rightarrow \mathbb{R}$ is sub-additive with respect to $T$ if

$$
\varphi_{m+n}(y) \leq \varphi_{m}\left(T^{n}(y)\right)+\varphi_{n}(y) \quad \forall y \in Y, \forall m, n \in \mathbb{N} .
$$

$\left\{\varphi_{n}\right\}_{n \in \mathbb{N}}$ is super-additive if $\left\{-\varphi_{n}\right\}_{n \in \mathbb{N}}$ is sub-additive.

Theorem 1.4. Let $\Phi$ be an SPT on $\Omega \times X$ with the base $\phi$ on $\Omega$ fulfilling $\left(H_{1}\right)$. Suppose that $a \in \mathbb{R}$ and $\left\{\varphi_{n}\right\}_{n \in \mathbb{N}} \subset C(\Omega \times X)$ is a sub-additive sequence with respect to $\Phi$ such that for every ergodic measure $\lambda \in \mathcal{M}(\Omega \times X, \Phi)$, there exists some $m \in \mathbb{N}$ such that

$$
\frac{1}{m} \int_{\Omega \times X} \varphi_{m} \mathrm{~d} \lambda \leq a .
$$

Then, for any given $\varepsilon>0$, there exists some $N_{\varepsilon} \in \mathbb{N}$ such that for all $n \geq N_{\varepsilon}$, there holds

$$
\frac{1}{n} \varphi_{n}(\omega, x) \leq a+\varepsilon \quad \forall(\omega, x) \in \Omega \times X
$$

Note that the statement is reasonable, because for an SPT $\Phi$ satisfying hypothesis $\left(H_{1}\right), \Phi$ must have some ergodic Borel probability measure. See Theorem 2.5. The proof of Theorem 1.4 will be given in Section 4 Although condition (1.7) is mathematical to some extent, it can be used to deduce the uniformly expanding property of a $C^{1}$ local diffeomorphism [1, 3] and to study the strange non-chaotic attractor [13].

\section{Some Properties about inVARiant measures of SPTs}

Let us first recall from [14, 15, some basic facts in measure theory. Suppose that $Y$ is a compact metric space. The Borel $\sigma$-algebra of $Y$ is denoted by $\mathcal{B}(Y)$. The set of all probability measures defined on the measurable space $(Y, \mathcal{B}(Y))$ is denoted by $\mathcal{M}(Y)$. The space of all real-valued continuous functions on $Y$ is denoted by $C(Y)$. Endowed with the supremum norm $\|\cdot\|_{\infty},\left(C(Y),\|\cdot\|_{\infty}\right)$ is a Banach space. By the Riesz representation theorem [14, Theorem 6.3], we know that there exists a bijection between $\mathcal{M}(Y)$ and the set of all normalised positive linear functionals on $C(Y)$. Therefore, $\mathcal{M}(Y)$ is identified with a convex subset of the unit ball in $C(Y)^{*}$, the dual space of $C(Y)$. Endowed with the weak* topology on $C(Y)^{*}$, we know that $\left(\mathcal{M}(Y), w^{*}\right)$ is convex and sequentially compact [14, Theorem 6.5].

A measurable mapping $T:(Y, \mathcal{B}(Y)) \rightarrow(Y, \mathcal{B}(Y))$ can yield a push $T_{*}: \mathcal{M}(Y) \rightarrow$ $\mathcal{M}(Y)$ by

$$
T_{*} \mu(B):=\mu\left(T^{-1}(B)\right) \quad \forall B \in \mathcal{B}(Y), \mu \in \mathcal{M}(Y) .
$$

A point $y \in Y$ can determine a measure defined by

$$
\delta_{y}(B):=\left\{\begin{array}{ll}
1 & \text { if } y \in B \\
0 & \text { if } y \notin B
\end{array} \quad \forall B \in \mathcal{B}(Y) .\right.
$$

Denote the discontinuity set by

$$
D_{T}:=\{y \in Y: T \text { is discontinuous at } y\} .
$$

Lemma 2.1 ([17). For any mapping $T: Y \rightarrow Y, D_{T}$ is an $F_{\sigma}$-set.

It is well-known that if $T$ is continuous, i.e., $D_{T}=\emptyset$, then so is the push $T_{*}$. When $T$ is only measurable, $T_{*}$ is in general not continuous in weak ${ }^{*}$ topology at all measures. However, with some restriction on measures, we have the following continuity result for $T_{*}$. 
Lemma 2.2 ([15, p. 171]). Suppose that $T:(Y, \mathcal{B}(Y)) \rightarrow(Y, \mathcal{B}(Y))$ is measurable. For any sequence $\mu_{n} \rightarrow \mu_{0}$ in $\left(\mathcal{M}(Y), w^{*}\right)$ satisfying $\mu_{0}\left(D_{T}\right)=0$, one has $T_{*} \mu_{n} \rightarrow$ $T_{*} \mu_{0}$ in $\left(\mathcal{M}(Y), w^{*}\right)$.

With this result at hand, by applying the Schauder-Tychonoff fixed point theorem [5. p. 456], we can obtain the existence of invariant Borel probability measures for SPTs. For more details of the proof, see [17.

Theorem 2.3. Let $\Phi$ be an SPT on $\Omega \times X$ with the base $\phi$ on $\Omega$. Suppose that $\nu\left(\pi\left(D_{\Phi}\right)\right)=0 \quad$ for some $\nu \in \mathcal{M}(\Omega, \phi)$.

Then $\Phi$ admits at least one invariant Borel probability measure, i.e., $\mathcal{M}(\Omega \times X, \Phi) \neq$ $\emptyset$.

Since $\pi: \Omega \times X \rightarrow \Omega$ is a closed mapping, we have from Lemma 2.1 that $\pi\left(D_{\Phi}\right)$ is an $F_{\sigma}$-set as well. Thus it implies that $\pi\left(D_{\Phi}\right) \in \mathcal{B}(\Omega)$. Note that $\left(H_{0}\right)$ is weaker than $\left(H_{1}\right)$.

Some further properties about $\mathcal{M}(\Omega \times X, \Phi)$ are listed as follows.

Lemma 2.4. Let $\Phi$ be an SPT on $\Omega \times X$ with the base $\phi$ on $\Omega$ fulfilling $\left(H_{1}\right)$. Then

(i) $\mathcal{M}(\Omega \times X, \Phi)$ is a compact convex subset of $\mathcal{M}(\Omega \times X)$.

(ii) $\mu$ is an extreme point of $\mathcal{M}(\Omega \times X, \Phi)$ if and only if $\mu$ is ergodic under $\Phi$.

Proof. (i) The convexity of $\mathcal{M}(\Omega \times X, \Phi)$ is clear from the linearity of push $T_{*}$. Let $\mu_{0} \in \mathcal{M}(\Omega \times X)$ and $\left\{\mu_{n}\right\}_{n \in \mathbb{N}} \subset \mathcal{M}(\Omega \times X, \Phi)$ be such that

$$
\mu_{n} \rightarrow \mu_{0} \quad \text { in }\left(\mathcal{M}(\Omega \times X), w^{*}\right) .
$$

It suffices to show $\Phi_{*} \mu_{0}=\mu_{0}$, because $\mathcal{M}(\Omega \times X)$ is compact.

At first, we claim that

$$
\pi_{*} \mu_{0} \in \mathcal{M}(\Omega, \phi) .
$$

To this end, we have, for $n \in \mathbb{N}$,

$$
\begin{aligned}
\phi_{*}\left(\pi_{*} \mu_{n}\right) & =(\phi \circ \pi)_{*} \mu_{n} \\
& =(\pi \circ \Phi)_{*} \mu_{n} \quad \text { (by definition of SPT) } \\
& =\pi_{*}\left(\Phi_{*} \mu_{n}\right) \\
& \left.=\pi_{*} \mu_{n} \quad \text { (because } \mu_{n} \in \mathcal{M}(\Omega \times X, \Phi)\right) .
\end{aligned}
$$

This implies that $\pi_{*} \mu_{n} \in \mathcal{M}(\Omega, \phi)$. Note that $\pi: \Omega \times X \rightarrow \Omega$ is continuous. Then by (2.2) one has

$$
\pi_{*} \mu_{n} \rightarrow \pi_{*} \mu_{0} \quad \text { in }\left(\mathcal{M}(\Omega), w^{*}\right) .
$$

Since $\mathcal{M}(\Omega, \phi) \subset \mathcal{M}(\Omega)$ is compact, we have the desired result (2.3).

Next, it follows from hypothesis $\left(H_{1}\right)$ that

$$
\mu_{0}\left(D_{\Phi}\right) \leq \mu_{0}\left(\pi^{-1}\left(\pi\left(D_{\Phi}\right)\right)\right)=\pi_{*} \mu_{0}\left(\pi\left(D_{\Phi}\right)\right)=0 .
$$

Combining with Lemma 2.2. we have

$$
\Phi_{*} \mu_{n} \rightarrow \Phi_{*} \mu_{0} .
$$

Note that $\Phi_{*} \mu_{n}=\mu_{n}$. Then (2.2) and (2.4) lead to $\Phi_{*} \mu_{0}=\mu_{0}$, finishing this part of the proof.

(ii) We can use the similar argument in [14, Theorem 6.10] to get the desired result. 
Theorem 2.5. Let $\Phi$ be an SPT on $\Omega \times X$ with the base $\phi$ on $\Omega$ fulfilling $\left(H_{1}\right)$. Then there exists at least one ergodic Borel probability measure under $\Phi$.

Proof. Since any non-empty compact convex set in a Banach space has extreme points [5, p. 439], the theorem is clear from Lemma 2.4

\section{Additive CASe}

Proof of Theorem 1.2. Assume that (1.3) is false. Then there exist $\varepsilon_{0}>0, n_{k} \uparrow \infty$, and $\left(\omega_{k}, x_{k}\right) \in \Omega \times X$ such that

$$
\frac{1}{n_{k}} \sum_{i=0}^{n_{k}-1} h\left(\Phi^{i}\left(\omega_{k}, x_{k}\right)\right)>a+\varepsilon_{0} \quad \forall k \in \mathbb{N} .
$$

For any $k \in \mathbb{N}$, by the Riesz representation theorem [14, Theorem 6.3], we know that three exists a measure $\mu_{k} \in \mathcal{M}(\Omega \times X)$ such that

$$
\frac{1}{n_{k}} \sum_{i=0}^{n_{k}-1} f\left(\Phi^{i}\left(\omega_{k}, x_{k}\right)\right)=\int_{\Omega \times X} f \mathrm{~d} \mu_{k} \quad \forall f \in C(\Omega \times X) .
$$

In fact, $\mu_{k}$ can be represented as

$$
\mu_{k}=\frac{1}{n_{k}} \sum_{i=0}^{n_{k}-1} \delta_{\Phi^{i}\left(\omega_{k}, x_{k}\right)} .
$$

Since $\mathcal{M}(\Omega \times X)$ is compact, we assume that there exists $\mu_{0} \in \mathcal{M}(\Omega \times X)$ such that

$$
\mu_{k} \rightarrow \mu_{0} \quad \text { in }\left(\mathcal{M}(\Omega \times X), w^{*}\right) .
$$

It means that, as $k \rightarrow \infty$,

$$
\frac{1}{n_{k}} \sum_{i=0}^{n_{k}-1} f\left(\Phi^{i}\left(\omega_{k}, x_{k}\right)\right)=\int_{\Omega \times X} f \mathrm{~d} \mu_{k} \rightarrow \int_{\Omega \times X} f \mathrm{~d} \mu_{0} \quad \forall f \in C(\Omega \times X) .
$$

Now we prove the following three assertions.

Assertion I. One has

$$
\pi_{*} \mu_{0} \in \mathcal{M}(\Omega, \phi) .
$$

In fact, given any $\tilde{f} \in C(\Omega)$, let us consider $f=\tilde{f} \circ \pi \in C(\Omega \times X)$. Then

$$
\begin{aligned}
\frac{1}{n_{k}} \sum_{i=0}^{n_{k}-1} \tilde{f}\left(\phi^{i}\left(\omega_{k}\right)\right) & =\frac{1}{n_{k}} \sum_{i=0}^{n_{k}-1} f\left(\Phi^{i}\left(\omega_{k}, x_{k}\right)\right) \\
& \rightarrow \int_{Y} f \mathrm{~d} \mu_{0} \quad(\text { by }(3.5)) \\
& =\int_{\Omega} \tilde{f} \mathrm{~d}\left(\pi_{*} \mu_{0}\right) .
\end{aligned}
$$

Since $\tilde{f} \in C(\Omega)$ is arbitrary, the Bogoliubov-Krylov process for the construction of invariant measures yields the desired result (3.6).

Assertion II. One has

$$
\Phi_{*} \mu_{k} \rightarrow \Phi_{*} \mu_{0} \quad \text { in }\left(\mathcal{M}(\Omega \times X), w^{*}\right) .
$$


Note that

$$
0 \leq \mu_{0}\left(D_{\Phi}\right) \leq\left(\pi_{*} \mu_{0}\right)\left(\pi\left(D_{\Phi}\right)\right)=0,
$$

where (3.6) and $\left(H_{1}\right)$ are used. Then assertion (3.7) can be easily deduced by (3.4) and Lemma 2.2.

Assertion III. One has

$$
\Phi_{*} \mu_{k} \rightarrow \mu_{0} \quad \text { in }\left(\mathcal{M}(\Omega \times X), w^{*}\right) .
$$

In fact, for any $f \in C(\Omega \times X)$, we have

$$
\begin{aligned}
\int_{\Omega \times X} f \mathrm{~d}\left(\Phi_{*} \mu_{k}\right) & =\int_{\Omega \times X} f \circ \Phi \mathrm{d} \mu_{k} \\
& \left.\left.=\frac{1}{n_{k}} \sum_{i=0}^{n_{k}-1} f \circ \Phi\left(\Phi^{i}\left(\omega_{k}, x_{k}\right)\right) \quad \text { (by (2.1) }\right) \text { and (3.3) }\right) \\
& \left.=\int_{\Omega \times X} f \mathrm{~d} \mu_{k}+\frac{1}{n_{k}}\left(f \circ \Phi^{n_{k}}\left(\omega_{k}, x_{k}\right)-f\left(\omega_{k}, x_{k}\right)\right) \quad \text { (by (3.2) }\right) .
\end{aligned}
$$

Combining with (3.4), we conclude that

$$
\lim _{k \rightarrow \infty} \int_{\Omega \times X} f \mathrm{~d} \Phi_{*} \mu_{k}=\int_{\Omega \times X} f \mathrm{~d} \mu_{0} .
$$

Since $f \in C(\Omega \times X)$ is arbitrary, assertion (3.8) is completed.

By (3.7) and (3.8), we have $\mu_{0} \in \mathcal{M}(\Omega \times X, \Phi)$. Finally, it follows from (3.1), (3.2) and (3.4) that

$$
\int_{\Omega \times X} h \mathrm{~d} \mu_{0}=\lim _{k \rightarrow \infty} \frac{1}{n_{k}} \sum_{i=0}^{n_{k}-1} h\left(\Phi^{i}\left(\omega_{k}, x_{k}\right)\right) \geq a+\varepsilon_{0},
$$

a contradiction to condition (1.2) of Theorem 1.2 ,

Let us point out that the sufficient condition (1.2) to guarantee the semi-uniform result (1.3) is also necessary. In fact, given any $\mu \in \mathcal{M}(\Omega \times X, \Phi)$, it follows from the Birkhoff ergodic theorem [14] that

$$
\lim _{n \rightarrow \infty} \frac{1}{n} \sum_{i=0}^{n-1} h\left(\Phi^{i}(\omega, x)\right)=h^{*}(\omega, x)
$$

exists for $\mu$-a.e. $(\omega, x) \in \Omega \times X$ and

$$
\int_{\Omega \times X} h^{*} \mathrm{~d} \mu=\int_{\Omega \times X} h \mathrm{~d} \mu .
$$

It follows from (1.3) and (3.9) that $h^{*}(\omega, x) \leq a+\varepsilon$ for $\mu$-a.e. $(\omega, x) \in \Omega \times X$. Then $\int_{\Omega \times X} h^{*} \mathrm{~d} \mu \leq a+\varepsilon$. Combining with (3.10), we have

$$
\int_{\Omega \times X} h \mathrm{~d} \mu \leq a+\varepsilon .
$$

Since $\varepsilon$ is arbitrary, (1.2) is necessarily satisfied.

Similarly, we have the following result. 
Theorem 3.1. Let $\Phi$ be an SPT on $\Omega \times X$ with the base $\phi$ on $\Omega$ fulfilling $\left(H_{1}\right)$. Suppose that $a \in \mathbb{R}$ and $h \in C(\Omega \times X)$. Then

$$
\int_{\Omega \times X} h \mathrm{~d} \mu \geq a \quad \forall \mu \in \mathcal{M}(\Omega \times X, \Phi)
$$

if and only if for any given $\varepsilon>0$, there exists some $N_{\varepsilon} \in \mathbb{N}$ such that for all $n \geq N_{\varepsilon}$ we have

$$
\frac{1}{n} \sum_{i=0}^{n-1} h\left(\Phi^{i}(\omega, x)\right) \geq a-\varepsilon \quad \forall(\omega, x) \in \Omega \times X .
$$

\section{Sub-ADDitive CASE}

In this section, $\Phi$ is always assumed to be an SPT on $\Omega \times X$ fulfilling $\left(H_{1}\right)$.

Note that in condition (1.7) of Theorem 1.4 the integer $m$ depends on the ergodic measure $\lambda \in \mathcal{M}(\Omega \times X, \Phi)$. However, this can be improved significantly.

Lemma 4.1. For $a \in \mathbb{R}$ and a sub-additive sequence $\left\{\varphi_{n}\right\}_{n \in \mathbb{N}} \subset C(\Omega \times X)$ with respect to $\Phi$, condition (1.7) implies that for any $\varepsilon>0$, there exists $m \in \mathbb{N}$ such that

$$
\frac{1}{m} \int_{\Omega \times X} \varphi_{m} \mathrm{~d} \mu \leq a+\varepsilon \quad \forall \mu \in \mathcal{M}(\Omega \times X, \Phi) .
$$

Proof. The proof is along the lines of Theorem 1.8 in [13. By sub-additivity we have

$$
\left|\frac{1}{n} \varphi_{n}(\omega, x)\right| \leq\left\|\varphi_{1}\right\|_{\infty} \quad \forall n \in \mathbb{N},(\omega, x) \in \Omega \times X
$$

By the Kingman sub-additive ergodic theorem [2, 9], for any $\mu \in \mathcal{M}(\Omega \times X, \Phi)$ we have

$$
\lim _{n \rightarrow \infty} \frac{1}{n} \varphi_{n}(\omega, x)=\bar{\varphi}(\omega, x) \quad \text { for } \mu \text {-a.e. }(\omega, x) \in \Omega \times X .
$$

Hence the Lebesgue Dominated Convergence Theorem shows that

$$
\lim _{n \rightarrow \infty} \frac{1}{n} \int_{\Omega \times X} \varphi_{n} \mathrm{~d} \mu=\int_{\Omega \times X} \bar{\varphi} \mathrm{d} \mu .
$$

Denote the set of all ergodic Borel probability measures under $\Phi$ by $\mathcal{E}(\Omega \times X, \Phi)$. Assume that $\lambda \in \mathcal{E}(\Omega \times X, \Phi)$. Then $\bar{\varphi}(\omega, x)$ is $\lambda$-a.e. constant, again denoted by $\bar{\varphi}$. Since

$$
\left\{\int_{\Omega \times X} \varphi_{n} \mathrm{~d} \lambda\right\}_{n \in \mathbb{N}}
$$

is a sub-additive real sequence, we know that

$$
\bar{\varphi}=\int_{\Omega \times X} \bar{\varphi} \mathrm{d} \lambda \leq \frac{1}{n} \int_{\Omega \times X} \varphi_{n} \mathrm{~d} \lambda \quad \forall n \in \mathbb{N} .
$$

Define the essential supremum $\varphi_{\mu}$ of $\bar{\varphi}$ with respect to $\mu$ by

$$
\varphi_{\mu}:=\inf \{M \in \mathbb{R} \mid \bar{\varphi}(\omega, x) \leq M \text { for } \mu \text {-a.e. }(\omega, x)\} .
$$

Then combining (1.7), (4.2) and (4.3), we conclude that

$$
\varphi_{\lambda} \leq a \quad \forall \lambda \in \mathcal{E}(\Omega \times X, \Phi) .
$$

Now we claim that

$$
\varphi_{\mu} \leq a \quad \forall \mu \in \mathcal{M}(\Omega \times X, \Phi) .
$$


In fact, it suffices to show that

$$
\mu[\bar{\varphi}(\omega, x)>a]=0 \quad \forall \mu \in \mathcal{M}(\Omega \times X, \Phi) .
$$

By the ergodic decomposition theorem [14, p. 153], for any $\mu \in \mathcal{M}(\Omega \times X, \Phi)$ there exists a probability measure $\tau$ on $\mathcal{E}(\Omega \times X, \Phi)$ such that

$$
\mu[\bar{\varphi}(\omega, x)>a]=\int_{\mathcal{E}(\Omega \times X, \Phi)} \lambda[\bar{\varphi}(\omega, x)>a] \mathrm{d} \tau(\lambda)=0,
$$

where (4.4) is used. Thus the desired result (4.6) is proved.

By (4.1), (4.3) and (4.5), we know that for any $\varepsilon>0$, there exists $N(\mu, \varepsilon) \in \mathbb{N}$ such that

$$
\frac{1}{n} \int_{\Omega \times X} \varphi_{n} \mathrm{~d} \mu \leq a+\varepsilon \quad \forall n \geq N(\mu, \varepsilon) .
$$

Note that for any $f \in C(\Omega \times X)$, the functional

$$
J_{f}(\mu):=\int_{\Omega \times X} f \mathrm{~d} \mu
$$

is continuous in $\mu \in\left(\mathcal{M}(\Omega \times X, \Phi)\right.$, $\left.w^{*}\right)$. Hence for any $\mu \in \mathcal{M}(\Omega \times X, \Phi)$, there exists an open neighborhood $\mathcal{O}(\mu, \varepsilon)$ of $\mu$ such that

$$
\frac{1}{N(\mu, \varepsilon)} \int_{\Omega \times X} \varphi_{N(\mu, \varepsilon)} \mathrm{d} \mu^{\prime} \leq a+2 \varepsilon \quad \forall \mu^{\prime} \in \mathcal{O}(\mu, \varepsilon),
$$

where (4.7) is used. The collection of all such $\mathcal{O}(\mu, \varepsilon)$ is an open cover of $\mathcal{M}(\Omega \times$ $X, \Phi)$. Since $\mathcal{M}(\Omega \times X, \Phi)$ is compact, we can find a finite subcover denoted by $\mathcal{O}\left(\mu_{1}, \varepsilon\right), \cdots, \mathcal{O}\left(\mu_{\ell}, \varepsilon\right)$. Thus for any $\mu \in \mathcal{M}(\Omega \times X, \Phi)$, we have $\mu \in \mathcal{O}\left(\mu_{j}, \varepsilon\right)$ for some $1 \leq j \leq \ell$. Furthermore, by (4.8) we obtain

$$
\frac{1}{N_{j}} \int_{\Omega \times X} \varphi_{N_{j}} \mathrm{~d} \mu \leq a+2 \varepsilon,
$$

where $N_{j}=N\left(\mu_{j}, \varepsilon\right)$. Let $k, n \in \mathbb{N}$. By sub-additivity again, we have

$$
\varphi_{k n}(\omega, x) \leq \sum_{i=0}^{k-1} \varphi_{n}\left(\Phi^{i n}(\omega, x)\right) \quad \forall(\omega, x) \in \Omega \times X .
$$

Then setting $n=N_{j}$, we obtain

$$
\begin{aligned}
\int_{\Omega \times X} \varphi_{k N_{j}} \mathrm{~d} \mu & \leq \sum_{i=0}^{k-1} \int_{\Omega \times X} \varphi_{N_{j}} \circ \Phi^{i N_{j}} \mathrm{~d} \mu \\
& =\sum_{i=0}^{k-1} \int_{\Omega \times X} \varphi_{N_{j}} \mathrm{~d} \mu=k \int_{\Omega \times X} \varphi_{N_{j}} \mathrm{~d} \mu .
\end{aligned}
$$

It implies that

$$
\frac{1}{k N_{j}} \int_{\Omega \times X} \varphi_{k N_{j}} \mathrm{~d} \mu \leq \frac{1}{N_{j}} \int_{\Omega \times X} \varphi_{N_{j}} \mathrm{~d} \mu .
$$

Denote $N:=N_{1} \times \cdots \times N_{\ell}$. By (4.9) and (4.10) we obtain

$$
\frac{1}{N} \int_{\Omega \times X} \varphi_{N} \mathrm{~d} \mu \leq a+2 \varepsilon \quad \forall \mu \in \mathcal{M}(\Omega \times X, \Phi),
$$

finishing the proof. 
Lemma 4.2. Suppose that $a \in \mathbb{R}$ and $\left\{\varphi_{n}\right\}_{n \in \mathbb{N}}$ is a sub-additive sequence with respect to $\Phi$. If there exists some $m \in \mathbb{N}$ such that

$$
\frac{1}{m} \int_{\Omega \times X} \varphi_{m} \mathrm{~d} \mu \leq a \quad \forall \mu \in \mathcal{M}(\Omega \times X, \Phi),
$$

then one has the semi-uniform result (1.8).

Proof. Denote

$$
M=\max _{0 \leq i \leq m} \max _{(\omega, x) \in \Omega \times X} \varphi_{i}(\omega, x),
$$

where $m$ is as in (4.11) and $\varphi_{0}(\omega, x):=0$. Then for any $k \in \mathbb{N}$ and $0 \leq j \leq m-1$, by the sub-additivity we obtain

$$
\varphi_{k m}(\omega, x) \leq \varphi_{j}(\omega, x)+\sum_{i=0}^{k-2} \varphi_{m}\left(\Phi^{i m+j}(\omega, x)\right)+\varphi_{m-j}\left(\Phi^{(k-1) m+j}(\omega, x)\right) .
$$

Summing over $j=0,1, \cdots, m-1$, we have

$$
\begin{aligned}
m \varphi_{k m}(\omega, x) & \leq \sum_{j=0}^{m-1}\left(\varphi_{j}(\omega, x)+\varphi_{m-j}\left(\Phi^{(k-1) m+j}(\omega, x)\right)\right)+\sum_{j=0}^{m-1} \sum_{i=0}^{k-2} \varphi_{m}\left(\Phi^{i m+j}(\omega, x)\right) \\
& =\sum_{j=0}^{m-1}\left(\varphi_{j}(\omega, x)+\varphi_{m-j}\left(\Phi^{(k-1) m+j}(\omega, x)\right)\right)+\sum_{j=0}^{(k-1) m-1} \varphi_{m}\left(\Phi^{j}(\omega, x)\right) \\
& \leq 2 m M+\sum_{j=0}^{(k-1) m-1} \varphi_{m}\left(\Phi^{j}(\omega, x)\right) .
\end{aligned}
$$

It implies that

$$
\varphi_{k m}(\omega, x) \leq 2 M+\sum_{j=0}^{(k-1) m-1} \frac{1}{m} \varphi_{m}\left(\Phi^{j}(\omega, x)\right) .
$$

By Theorem 1.2 we have that for any given $\varepsilon>0$, there exists $L \in \mathbb{N}$ such that for all $\ell \geq L$ we have

$$
\frac{1}{\ell} \sum_{j=0}^{\ell-1} \frac{1}{m} \varphi_{m}\left(\Phi^{j}(\omega, x)\right) \leq a+\varepsilon .
$$

It follows from (4.12) and (4.13) that for all $k \in \mathbb{N}$ such that $(k-1) m-1 \geq L$, there holds

$$
\begin{aligned}
\varphi_{k m}(\omega, x) & \leq 2 M+(k-1) m(a+\varepsilon) \\
& \leq 2 M+(k-1) m a+(k-1) m \varepsilon .
\end{aligned}
$$

Now for any $n \geq L+2 m$, denote $n=k m+j, 0 \leq j \leq m-1$. Then

$$
(k-1) m-1=n-j-m-1 \geq L+2 m-j-m-1 \geq L .
$$

By sub-additivity again, for any $n \geq L+2 m$, we obtain

$$
\begin{aligned}
\varphi_{n}(\omega, x) & \leq \varphi_{k m}(\omega, x)+\varphi_{j}\left(\Phi^{k m}(\omega, x)\right) \\
& \leq \varphi_{k m}(\omega, x)+M .
\end{aligned}
$$

Combining (4.14), (4.15) and (4.16), we have

$$
\varphi_{n}(\omega, x) \leq(k-1) m a+(k-1) m \varepsilon+3 M \quad \text { when } n \geq L+2 m .
$$


Dividing by $n$, we obtain

$$
\frac{1}{n} \varphi_{n}(\omega, x) \leq \frac{(k-1) m a}{n}+\frac{(k-1) m \varepsilon}{n}+\frac{3 M}{n} .
$$

Note that $(k-1) m \leq n$. Then

$$
\frac{1}{n} \varphi_{n}(\omega, x) \leq a+\varepsilon+\frac{3 M}{n} .
$$

Therefore, for any $n \geq \max \{L+2 m, 3 M / \varepsilon\}$, we have

$$
\frac{1}{n} \varphi_{n}(\omega, x) \leq a+2 \varepsilon,
$$

completing the proof.

Proof of Theorem 1.4. It is an immediate result of Lemma 4.1 and Lemma 4.2 .

Note that the sufficient condition (1.7) to guarantee the semi-uniform result (1.8) is also necessary. Indeed, let $\lambda$ be any ergodic measure. By the Kingman sub-additive ergodic theorem [2, 9], we have

$$
\lim _{n \rightarrow \infty} \frac{1}{n} \varphi_{n}(\omega, x)=\bar{\varphi} \quad \text { for } \lambda \text {-a.e. }(\omega, x) \in \Omega \times X,
$$

where $\bar{\varphi}$ is constant. By (1.7), we have $\bar{\varphi} \leq a+\varepsilon$. Since $\varepsilon$ is arbitrary, we get $\bar{\varphi} \leq a$. Now it follows from sub-additivity of $\left\{\varphi_{n}\right\}$ and the Lebesgue Dominated Convergence Theorem that

$$
\inf _{n \in \mathbb{N}}\left\{\frac{1}{n} \int_{\Omega \times X} \varphi_{n} \mathrm{~d} \lambda\right\}=\lim _{n \rightarrow \infty} \frac{1}{n} \int_{\Omega \times X} \varphi_{n} \mathrm{~d} \lambda=\bar{\varphi} \leq a .
$$

Therefore we get the desired result (1.7).

We end the paper with some remarks.

At first, compared with Theorem 1.19 and Theorem 1.20 in 13 where the semiuniform result is obtained only for $x \in X$ when $\omega$ is fixed, our semi-uniform results for all $(\omega, x) \in \Omega \times X$ are much stronger. Moreover, the discontinuity of $\Phi$ is allowed in our results.

Second, taking this opportunity, we would like to make a modification in Theorem 2.6 in 18 . That is, the sequence of continuous functions $\varphi_{n}: X \rightarrow \mathbb{R}$ should be super-additive.

It is reasonably expected that, based on the results of this paper, some consequences in [13, 18, can be improved significantly. In fact, the forced systems even with phase transitions can be studied using the present results.

\section{ACKNOWLEDGMENTS}

The research of the first author is supported by the National Basic Research Program of China (grant No. 2006CB805903), 111 Project of China (2007) and the Doctoral Fund of Ministry of Education of China (grant No. 20090002110079). The research of the second author is supported by the National Natural Science Foundation of China (grant No. 11071238) and the Key Lab of Random Complex Structures and Data Science, CAS. The research of the third author is supported by the China Postdoctoral Science Foundation (grant No. 2011M500414 and grant No. 2012T50146). 


\section{REFERENCES}

[1] J. Alves, V. Araujo and B. Saussol, On the uniform hyperbolicity of some nonuniformly hyperbolic systems, Proc. Amer. Math. Soc. 131 (2003), 1303-1309. MR.1948124 (2003k:37046)

[2] A. Avila and J. Bochi, On the subadditive ergodic theorem, Preprint, 2009.

[3] Y. Cao, Non-zero Lyapunov exponents and uniform hyperbolicity, Nonlinearity 16 (2003), 1473-1479. MR:1986306 (2005g:37061)

[4] X. Dai, Rotation number of forced set-valued maps of unit circle, Preprint, 2011.

[5] N. Dunford and J. T. Schwartz, Linear Operators, Part I, Interscience Publishers, New York, 1958. MR0117523 (22:8302)

[6] M. R. Herman, Une méthode pour minorer les exposants de Lyapunov et quelques exemples montrant le caractère local d'un théorème d'Arnol'd et de Moser sur le tore de dimension 2, Comment. Math. Helv. 58 (1983), 453-502. MR727713 (85g:58057)

[7] R. Johnson and J. Moser, The rotation number for almost periodic potentials, Comm. Math. Phys. 84 (1982), 403-438. Erratum, Comm. Math. Phys. 90 (1983), 317-318. MR667409 (83h:34018) MR0714441 (85a:34032)

[8] A. Katok and B. Hasselblatt, Introduction to the Modern Theory of Dynamical Systems, Cambridge Univ. Press, Cambridge, 1995. MR1326374 (96c:58055)

[9] J. F. C. Kingman, Subadditive ergodic theory, Ann. Prob. 1 (1973), 883-909. MR.0356192 (50:8663)

[10] H. Niikuni, The rotation number for the generalized Kronig-Penney Hamiltonians, Ann. Henri Poincaré 8 (2007), 1279-1301. MR2360437 (2008m:81049)

[11] J. C. Oxtoby, Ergodic sets, Bull. Amer. Math. Soc. 58 (1952), 116-136. MR.0047262 $(13: 850 \mathrm{e})$

[12] J. Stark, U. Feudel, P. A. Glendinning and A. Pikovsky, Rotation numbers for quasiperiodically forced monotone circle maps, Dyn. Syst. 17 (2002), 1-28. MR1888695 (2003h:37058)

[13] R. Sturman and J. Stark, Semi-uniform ergodic theorems and applications to forced systems, Nonlinearity 13 (2000), 113-143. MR 1734626 (2000m:37041)

[14] P. Walters, An Introduction to Ergodic Theory, Springer-Verlag, New York/Berlin, 1982. MR.648108(84e:28017)

[15] J. Yan, Lectures on Measure Theory, Science Press, Beijing, 2004 (in Chinese).

[16] M. Zhang and Z. Zhou, Rotation numbers of linear Schrödinger equations with almost periodic potentials and phase transmissions, Ann. Henri Poincaré 11 (2010), 765-780. MR2677743 (2011j:47139)

[17] M. Zhang and Z. Zhou, Uniform ergodic theorems for discontinuous skew-product flows and applications to Schrödinger equations, Nonlinearity 24 (2011), 1539-1564. MR2785981

[18] Z.-H. Zheng, J. Xia and Z. Zheng, Necessary and sufficient conditions for semi-uniform ergodic theorems and their applications, Discrete Contin. Dyn. Syst. 14 (2006), 409-417. MR2171719(2006g:37041)

Department of Mathematical Sciences, Tsinghua University, Beijing 100084, People's Republic of China

E-mail address: mzhang@math.tsinghua.edu.cn

Institute of Applied Mathematics, Academy of Mathematics and System Sciences, Chinese Academy of Sciences, Beijing 100190, People's Republic of China

E-mail address: zhzheng@amt.ac.cn

Institute of Applied Mathematics, Academy of Mathematics and System Sciences, Chinese Academy of Sciences, Beijing 100190, People's Republic of China

E-mail address: zzhou@amss.ac.cn 